\title{
Determination and characterization of total thiols in mouse serum samples using hydrophilic interaction liquid chromatography with fluorescence detection and mass spectrometry
}

\author{
Muneki Isokawa ${ }^{l}$, Tatsuo Shimosawa ${ }^{2}$, Takashi Funatsu ${ }^{l}$, Makoto Tsunoda ${ }^{l}$ * \\ ${ }^{1}$ Graduate School of Pharmaceutical Sciences, University of Tokyo \\ ${ }^{2}$ Graduate School of Medicine, University of Tokyo \\ 7-3-1 Hongo, Bunkyo-ku, Tokyo, 113-0033, Japan \\ *: corresponding author \\ E-mail: makotot@mol.f.u-tokyo.ac.jp \\ Fax: +81-3-5802-3339
}




\begin{abstract}
Biothiols such as homocysteine, cysteine, and glutathione play many biologically important roles, especially in reduction-oxidation homeostasisand resistance to oxidative stress, and the measurement of their concentrations in model animal fluids is important in clarifying the pathology of thiol-related diseases. In this study, an analytical method for total biothiols in mouse serum using hydrophilic interaction liquid chromatography (HILIC) with fluorescence detection was developed. Mouse serum samples were derivatized with ammonium7-fluoro-2,1,3-benzoxadiazole-4-sulfonate (SBD-F), after reduction by tris(2-carboxyethyl)phosphine. Five biothiols (homocysteine, cysteine, cysteinylglycine, glutathione, and $\gamma$-glutamylcysteine) in the mouse sera were separated within 16 min on an amide-type HILIC column. The method possessed good linearity, good reproducibility with an intra-day variance of less than 3\%, and low detection limits of $0.2-4 \mathrm{nM}$. Concentrations of homocysteine, cysteine, cysteinylglycine, glutathione, and $\gamma$-glutamylcysteine in the mouse serum samples were calculated as $6.7 \pm 0.3,227.7 \pm 16.9,1.2 \pm 0.4,77.5 \pm 29.2$, and $8.2 \pm 0.9 \mu \mathrm{M}$, respectively (mean \pm S.D., $n=4$ ). Furthermore, HILIC-negative electrospray ionization-mass spectrometry(MS) analysis using a high-resolution mass spectrometer was conducted to determine the exact masses of two unknown peaks, which were found in the mouse serum samples with high signal intensity and were not detected in human plasma samples. The exact masses of the unknown compounds were determined as 1184.519 and 800.281 (as SBD-derivatized negative ions),which possessed a product ion common to SBD-thiols $\left.(\mathrm{m} / \mathrm{z} \text { 230.954, as[SBD-SH] }]^{-}\right)$upon tandem MS spectrometric analysis.
\end{abstract}

Key words

Amide column, oxidative stress, SBD-F, thiol metabolism 


\section{Introduction}

Low-molecular-weight thiols such as cysteine (Cys), homocysteine (Hcy), and glutathione (GSH) are metabolites of the sulfur cycle in biological systems.Figure 1A shows representative endogenous thiols related to the metabolism of Cys and GSH, which are abundant reductants in our bodies[1]. In a biological system,Hcy can be synthesized from methionine[2], and converted to Cys[3]. GSH is synthesized from $\gamma$-glutamylcysteine ( $\gamma$ GluCys)[4], and the degradation of GSH leads to the formation of cysteinylglycine (CysGly)[5]. The $N$-acetylation of Cys residues produces $\mathrm{N}$-acetylcysteine (NAC)[6]. These biothiols play important roles in many biological processes, including the maintenance of reduction-oxidation (redox) homeostasisagainst oxidative stress and the inactivation of toxic compounds[1, 7-10]. In addition, imbalances inbiothiol metabolism areclosely related to various pathologies such as vascular diseases[11, 12], neurodegenerative diseases including Alzheimer's[13] and Parkinson's[14], cancers[15, 16], and diabetes mellitus[17, 18]. Therefore, the simultaneous determination of multiple biothiols in biological samples is important for clarifying the relationship between the in vivo redox status and these disease states.

Among the analytical methods for thiols, HPLC with fluorescence detection under reversed-phase liquid chromatography (RPLC) conditions has been the most widely used[19-23]. However, the methods adopting RPLC have been unable to determine all the GSH-related biothiols described above the retention and separation of highly polar, derivatized biothiols were difficult and the detection sensitivity was insufficient. Thus, only four biothiols, including Hcy, Cys, CysGly, and GSH, were quantified in most cases[7, 19-21]. To tackle this problem, we recently developed an analytical method for biothiols using hydrophilic interaction liquid chromatography (HILIC) as the separation mode and a widely used thiol-selective fluorogenic reagent, ammonium 7-fluoro-2,1,3-benzoxadiazole-4-sulfonate (SBD-F) (Fig. 1B)[24]. Because polar compounds are easily retained and separated under HILIC conditions[24-26], this method was capable of separating all the GSH-related biothiols, as well as cysteamine and $N$-(2-mercaptopropionyl)glycine (MPG) as an internal standard. Furthermore, the detection sensitivity was increased over RPLC methods due, in 
part, to the fluorescence enhancement of the SBD-thiols in the acetonitrile-rich HILIC mobile phase. Thus, six biothiols (NAC, Hcy, Cys, CysGly, GSH, and $\gamma$ GluCys) could be determined in a human plasma sample.The simultaneous determination of these GSH-related biothiols, including $\gamma$ GluCys, should be quite important for monitoringany changes in the biothiol balance, which could reflect oxidative stress in the body, since $\gamma$ GluCys is a precursor of the particularly important reductant, GSH. However, the method was applied to human- rather than the animal-based samples that are widely used in preclinical research. Mice, in particular, arefrequently employed animalmodels for biothiol-related studies, including those related tohyperhomocysteinemia[27],thiol- and vitamin B-related nutrition[28], and oxidative stress[29].

Hence, in this study, we sought to develop an analyticalmethod using HILIC and SBD-F thatcould be appliedto mouse serum samples. Herein, we report the successful quantification of total Hcy, Cys, CysGly, GSH, and $\gamma$ GluCys, and their separation from two unknown peaks in the mouse sera. Optimization of the separation conditions was conducted using an amide-typecolumn (Inertsil Amide)instead of the sulfoalkylbetaine-based column (ZIC-HILIC) used in the previous report, since, on the latter, numerous interference peaks were found in the mouse serum samples which were not observed for the human plasma samples. Furthermore, the exact masses of the unknown biothiols were determined using HILIC-quadrupole-Orbitrap-mass spectrometry (q-Orbitrap-MS). 


\section{Materials and methods}

\subsection{Chemicals and reagents}

L-Cysteine (Cys), DL-homocysteine (Hcy), L-glutathione (reduced form, GSH), cysteinylglycine (CysGly), and $\gamma$-glutamylcysteine ( $\gamma$ GluCys) were purchased from Sigma-Aldrich (St. Louis, MO, USA). N-Acetyl-L-cysteine (NAC), tiopronin ( $N$-(2-mercaptopropionyl)glycine, MPG), and trichloroacetic acid (TCA) were obtained from Wako (Osaka, Japan). Ammonium 7-fluoro-2,1,3-benzoxadiazole-4-sulfonate (SBD-F) was purchased from Dojindo (Kumamoto, Japan). Tris(2-carboxyethyl)phosphine (TCEP) was obtained from TCI (Tokyo, Japan). Water was purified using a Milli-Q system (Millipore, Bedford, MA, USA). HPLC-grade acetonitrile was used. All other chemicals were of analytical-reagent grade.

\subsection{Collection of biological samples}

The mouse serum and plasma samples used in the validation experiments were prepared from 16-week-old male 129SV mice. Blood was collected into normal or ethylenediamine- $N, N, N^{\prime}, N^{\prime}$-tetraacetate (EDTA) tubes for the serum or plasma samples, respectively, and cooled immediately in icewater to avoid enzymatic degradation of the biothiols[7, 21]. Serum and plasma were separated by centrifugation at 3,000 rpm for $10 \mathrm{~min}$. Collected serum and plasma samples were stored at $-20^{\circ} \mathrm{C}$ until use. All of the animals received humane care in compliance with the National Research Council's criteria outlined in the "Guide for the Care and Use of Laboratory Animals" prepared by the US National Academy of Sciences and published by the US National Institutes of Health. The protocol was approved by the Ethics Committee on Animal Research of the University of Tokyo (Permit Number: P14-147). All efforts were made to minimize suffering. Mouse serum, plasma, and human plasma used in the other experiments were purchased from Sigma-Aldrich. 


\subsection{Preparation of standard and biological samples}

Sample preparation for the quantification of the total biothiols (reduced thiols + disulfides)was conducted based on our previous report[24]. To reduce the disulfides and protein-bound thiols, $25 \mu \mathrm{L}$ of a thiol standard solution, mouse serum, plasma, or human plasma was added to a mixture of TCEP solution $(120 \mathrm{~g} / \mathrm{L}$ in phosphate buffer, $5 \mu \mathrm{L})$ andMPG solution $(8 \mu \mathrm{M}$ in phosphate buffer, $25 \mu \mathrm{L})$ as the internal standard. The resulting mixture was incubated for $0.5 \mathrm{~h}$ at room temperature. For deproteinization, the sample was mixed with $25 \mu \mathrm{L}$ of a TCA solution, and then centrifuged at $15,000 \times g$ for $10 \mathrm{~min}$ at $4^{\circ} \mathrm{C}$.A $50 \mu \mathrm{L}$ aliquot of the supernatant was added to the derivatization solution containing $123 \mu \mathrm{L}$ of borate buffer $(125 \mathrm{mM}$, pH 9.5), $50 \mu \mathrm{L}$ of SBD-F solution $(3.0 \mathrm{~g} / \mathrm{L}$ in borate buffer), and $2 \mu \mathrm{L}$ of $5 \mathrm{M}$ sodium hydroxide. After incubation for $1 \mathrm{~h}$ at $60^{\circ} \mathrm{C}$, the solution was mixed with $25 \mu \mathrm{L}$ of $1 \mathrm{M}$ hydrochloric acid and cooled in ice to quench the reaction. The resulting solution was diluted 5 times with acetonitrile for injection to avoid deterioration of the peak shape[30, 31]. Five microliters of the sample were injected into the HPLC system for analysis.

\subsection{Instruments for HPLC-fluorescence detection and chromatographic conditions}

The HPLC system consisted of a pump (PU-2080 Plus, JASCO, Tokyo, Japan), a column oven (CO-1560, JASCO), and a fluorescence detector (RF-20A, Shimadzu, Kyoto, Japan). ZIC-HILIC (150 $\mathrm{mm} \times 2.1 \mathrm{~mm}$ i.d., $5 \mu \mathrm{m}$, Merck, Germany) and Inertsil Amide $(150 \mathrm{~mm} \times 3.0 \mathrm{~mm}$ i.d., $5 \mu \mathrm{m}$, GL Sciences, Tokyo, Japan) columns were used. The column temperature was set at $35^{\circ} \mathrm{C}$, and the SBD-thiols were detected by fluorescence with excitation and emission wavelengths of 375 and 510 $\mathrm{nm}$, respectively. The chromatograms were analyzed using Chromato-Pro ver. 5.00software (Run Time Corporation, Kanagawa, Japan). Under optimized conditions, a mobile phase consisting of acetonitrile-40 mMammonium formate buffer $(\mathrm{pH} 3.0)(75: 25, \mathrm{v} / \mathrm{v})$ was used.The flow rate was 0.4 $\mathrm{mL} / \mathrm{min}$. 


\subsection{Validation}

Eight standard concentrations of the thiols - covering the range of 5-750 nM for SBD-NAC, -Hcy and -CysGly; 46-6900 nM for SBD-Cys; 10-1500 nM for SBD-GSH; and 15-2250 nM for $\gamma$ GluCys - were injected as calibration samples. Limits of detection (LODs) at a signal-to-noise ratio $(\mathrm{S} / \mathrm{N})$ equal to 3 and limits of quantification (LOQs) at $\mathrm{S} / \mathrm{N}=10$ were calculated from the chromatograms of 5 nM SBD-NAC, -Hcy, and -CysGly, 46 nM SBD-Cys, 10 nM SBD-GSH, and 15 nM SBD- $\gamma$ GluCys. The recovery of the thiols in the mouse sera was assessed by spiking additional thiols at three different concentrations. The intra- and inter-day precisions were assessed by replicate analyses of the same mouse serum sample on the same day and on sequential days, respectively. In the recovery and reproducibility analyses, the samples were injected five times. The quantification was conducted using the relative peak height to SBD-MPG (internal standard).

\subsection{Instruments and conditions for LC-MS}

A Nexera X2 HPLC system (Shimadzu) and a Q-Exactive Plus mass spectrometer (Thermo Fisher Scientific Inc., Waltham, MA,USA) were employed for LC-MS. The system control and data analysis were conducted with Xcalibur 3.0.63 software (Thermo Fisher Scientific Inc.). The chromatographic conditions were the same as described in section 2.4. The q-Orbitrap ion source was a heated electrospray ionization source II (HESI II) probe, and HILIC-MS analysis was performed in the ESI negative mode. The MS parameters were as follows: spray voltage, $2.5 \mathrm{kV}$; capillary temperature, $270^{\circ} \mathrm{C}$; sheath gas flow rate, 50 [a.u.]; auxiliary gas flow rate, 15 [a.u.]; sweep gas flow rate, 3 [a.u.]; and S-lens RF level, 55 [a.u.]. To achieve full mass spectra, tandem mass (MS/MS) spectra, and selective ion monitoring (SIM) spectra, analytical modes corresponding to Full MS-SIM, targeted-MS ${ }^{2}$ (or parallel reaction monitoring (PRM)), and targeted-SIM (or t-SIM) were employed. MS/MS analyses were typically conducted with a collision energy of $30 \mathrm{eV}$. Nitrogen was used as the collision gas and damping gas. Elemental analysis based on the exact mass was carried out using Xcalibur Qual Browser software. 


\section{Results and discussion}

\subsection{Optimization of separation conditions for SBD-thiols in mouse sera}

The ZIC-HILIC column was first used for the separation of the SBD-thiols in the mouse serum samples, since this zwitterionic column was the best for SBD-thiol separation among six different HILIC columns used in our previous study[24]. However, interference peaks were observed inthe chromatograms of the mouse sera, resulting in a failure to separate the SBD-thiols. The Inertsil Amide column was the second best in terms of SBD-thiol retention and separation[24], and, hence, was selected to effect sufficient separation of the SBD-thiols in mouse sera.

Since two unknown peaks with high signal intensity (UK1 and UK2) were found in the chromatograms of the mouse serum samples with an initial mobile phase of acetonitrile-10 mM ammonium formate buffer $(\mathrm{pH} 3.0)(75: 25, \mathrm{v} / \mathrm{v})$, the concentration of the ammonium formate buffer in the mobile phase was investigated $(10,20,40,50$, and $100 \mathrm{mM})$ to obtain sufficient separation ofthe SBD-thiols from the unknown peaks. Figure 2 shows the buffer-concentration-dependent changes in retention times of the SBD-thiols and the two unknowns. Interestingly, the known SBD-thiols were more retained in the mobile phase with higher buffer concentration, whereas the unknowns showed the opposite trend. This may indicate that the unknown thiols have some different features from the known low-molecular-weight biothiols. The buffer concentration in the mobile phase was selected as $40 \mathrm{mM}$, at which the best separation was achieved.

Under these optimum conditions, the six SBD-biothiols, internal standard, and two unknown peaks were successfully separated in 16 min, as shown in Fig.3.Five biothiols including Hcy, Cys, CysGly, GSH, and $\gamma$ GluCys were detected in the mouse serum sample, whereas NAC was not found (Fig. 3B). The peak heights ofUK1 and UK2 were comparable to those of SBD-Hcy and -GSH, which are typically present at concentrations on the order of $10 \mu \mathrm{M}$ in mouse sera, as shown in Fig. 3B. UK1 and UK2 were considered to possess SBD-derivatized thiol groups, since the reaction products of SBD-F and functional groups other than thiol would not typically fluoresce. Although the fluorescence intensity of an SBD-thiol species usually depends on its chemical structure[32], the 
concentrations of the two unknown bio thiols in the mouse sera were considered comparable to the known bio thiols based on the comparison of their peak heights to those of the known SBD-thiols. In addition, UK1 and UK2 were not found in the mouse serum samples without the reduction treatment by TECP, whereas the five known bio thiols were detected. This indicates that the unknown bio thiols most likely exist in the disulfide forms in mouse blood.

The two large peaks noted for the mouse serum sample were not detectable in the case of the human plasma sample(Fig. 3C). Since the unknown peaks were detected in several batches of purchased mouse serum and plasma as well as in freshly collected mouse serum and plasma, we confirmed that UK1 and UK2 actually exist in mouse blood and are not artifacts. These results indicate that there is likely to be a difference in sulfur metabolism between mice and humans. Further investigations of UK1 and UK2 are described in Section 3.3.

\subsection{Validation of the developed method}

Table 1 shows the LODs, LOQs, and linearity of the method for determining the SBD-thiols. The LODs were comparable to the previous method that used SBD-F and the ZIC-HILIC column[24], and30-250 times lower than methods using SBD-F and RPLC[33-35].The increase in detection sensitivity under HILIC conditions over RPLC conditions is likely due to the fluorescence enhancement of the SBD-thiols in the acetonitrile-rich HILIC mobile phase[24]. The high detection sensitivity should enable application of this method in the determination of the reduced-form thiols in biological samples, in addition to total thiols determination. The concentrations of five biothiols (Hcy, Cys, CysGly, GSH, and $\gamma$ GluCys)in the mouse serum samples were determined as6.7 \pm 0.3 , $227.7 \pm 16.9,1.2 \pm 0.4,77.5 \pm 29.2$, and $8.2 \pm 0.9 \mu \mathrm{M}$, respectively (mean \pm S.D., $n=4$ ), and those in the mouse plasma samples were calculated as $5.8 \pm 0.8,179.3 \pm 7.5,1.3 \pm 0.4,80.2 \pm 40.9$, and $7.4 \pm 0.5 \mu \mathrm{M}$, respectively (mean \pm S.D., $n=3$ ). The calculated concentrations were in good agreement with a previous report (in mouse plasma sample: 12, 124, 3, and $35 \mu \mathrm{M}$ for Hcy, Cys, CysGly, and GSH, respectively)[36]. The developed method is advantageousin terms of 
understanding endogenous sulfur metabolism, becausethere are few analytical methods that can quantify $\gamma$ GluCys in biological fluids $[24,37,38]$. The quantification of $\gamma$ GluCys should provide essential information about the rolesof biothiols, especially in response to oxidative stress, since $\gamma$ GluCys is a precursor of the particularly important reductant, GSH. In addition, only this method could separate these GSH-related biothiols, as well as some unknown peaks.

Intra-day precision and recovery results are shown in Table 2. The coefficients of variation (CVs)of the intra-day assaysfor all biothiols were less than 3\%, and the recoveries were between 95 and $109 \%$. The CVs of the inter-day assays were lower than 4, 7, 15, 6, and 10\% for Hcy, Cys, CysGly, GSH, and $\gamma$ GluCys, respectively.

\subsection{Investigation of unknown thiolated compounds in mouse sera using HILIC-q-Orbitrap-MS analysis}

As described above, two unknown peaks (UK1 and UK2) with high signal intensity were found only in mouse serumsamples, and not in human plasma. To acquire more information about the unknown compounds, HILIC-MS and HILIC-MS/MS analyses were conducted. Q-Orbitrap-MS, which enables the measurement of the exact compound mass with a resolution of 70,000 , was employed as the MS detector.

Since this is the first report in which SBD-derivatized thiols were analyzed by HILIC-q-Oribtrap-MS,standard samples were initially analyzed. Using the ESI negative mode as an ionization method, $1 \mu \mathrm{M} \mathrm{SBD}$-thiols were detected with sufficient signal intensity. The exact masses of SBD-NAC, -Hcy, -Cys, -CysGly, -GSH, and - $\gamma$ GluCys were successfully determined as 359.997, 332.002, 317.986, 375.007, 504.050, and 447.029, respectively, in good agreement with the theoretical exact masses $(359.997,332.002,317.986,375.007,504.050$, and 447.029, respectively).Next, HILIC-MS/MS analyses were conducted. A common product ion $(\mathrm{m} / z$ 230.954)with the strongest intensity was found in the MS/MS spectra of all the SBD-thiols. It was estimated that this product ion must derive from the SBD-S moiety: namely,[SBD-SH] $]^{-}$, for which 
the theoretical exact mass is 230.954.Considering these results, we could therefore confirm that a precursor ion possessed the SBD-S moiety by judging the existence of the product ion of $\mathrm{m} / \mathrm{z}$ 230.954 .

To confirm the presence of thiols and to determine the exact masses of the unknown peaks, HILIC-MS and -MS/MS analyses were applied to the mouse serum samples. Figures 4A-E show the extracted MS chromatograms corresponding to each SBD-thiol. Five SBD-thiols (SBD-Hcy, -Cys, -CysGly, -GSH, and - $\gamma$ GluCys) were confirmed in the mouse serum samples. The assignments of the unknown compounds were carried out based on the following criteria:1) the ion peak in the extracted MS chromatogram must have the same retention time and peak shape as that in the fluorescence chromatogram; 2) the ion peak must exist only in the SBD-derivatized mouse serum sample, and not in the mouse serum sample prior to derivatization; and 3) the ion must possess the product ion $m / z$ 230.954, which confirms the SBD-S moiety, as mentioned above. Based on these criteria, the exact masses of UK1 and UK2 were estimated as 1184.519 and 800.281 , respectively. Itwas found that UK1 also existed as a divalent ion $(\mathrm{m} / \mathrm{z}$ 591.756). Peaks of these ions were not detected in the SBD-derivatized human plasma sample. The exact masses of UK1 and UK2 without the SBD moiety were 3 and 2 times larger than that of GSH, respectively. This large difference in molecular weights is probably responsible for the different trends in the retention time versus buffer concentration in the mobile phase between the known and unknown biothiols, as discussed above. The extracted MS chromatograms of the estimated exact mass are shown in Figs. 4F-H. Figure 5 shows the MS/MS spectra of UK1 (precursor ion: $\mathrm{m} / \mathrm{z}$ 591.756) and UK2 (precursor ion: $\mathrm{m} / \mathrm{z}$ 800.281); the product ion $m / z 230.954$ is present in both spectra. In addition, $\mathrm{m} / \mathrm{z} 952.557$ was found in the MS/MS spectrum of UK1, where the precursor ion was selected as its divalent ion (Fig. 5A). This ion is considered to be the UK1 residue after loss of the SBD-Smoiety $(952.557+230.954$ $\left.[\mathrm{SBD}-\mathrm{SH}]^{-}+1.008[\mathrm{H}]^{+}=1184.519[\mathrm{UK} 1]^{-}\right)$. From these results, we confirmed that the univalent ion with $\mathrm{m} / z 1184.519$ and divalent ion with $\mathrm{m} / \mathrm{z} 591.756$ must be from an identical thiol-containing compound. 
Finally, elemental analyses based on exact mass were carried out using Xcalibur Qual Browser software under the constraints in which the compounds must contain SBD-S moieties $(100 \geq \mathrm{C} \geq 6$, $150 \geq \mathrm{H} \geq 2,30 \geq \mathrm{O} \geq 4,30>\mathrm{N}>2,10 \geq \mathrm{S} \geq 2$ ) and double-bond equivalents (DBE; $100 \geq \mathrm{DBE} \geq 7$ ). In the cases of the known thiols, the correct formulas were successfully suggested as the most or the second-most possible formulas by the software. Under those calculation conditions, the elemental formulas of UK1 and UK2 were also predicted. It was difficult to determine the correct elemental formulas, since large numbers were suggested (i.e., $>30$ with 5 ppm mass tolerances in both cases), owing to their high molecular weights. The top five formulas in terms of mass tolerance were $\mathrm{C}_{48} \mathrm{H}_{92} \mathrm{O}_{4} \mathrm{~N}_{14} \mathrm{~S}_{8}, \mathrm{C}_{46} \mathrm{H}_{66} \mathrm{O}_{6} \mathrm{~N}_{29} \mathrm{~S}_{2}, \mathrm{C}_{47} \mathrm{H}_{72} \mathrm{O}_{11} \mathrm{~N}_{22} \mathrm{~S}_{2}, \mathrm{C}_{48} \mathrm{H}_{78} \mathrm{O}_{16} \mathrm{~N}_{15} \mathrm{~S}_{2}$, and $\mathrm{C}_{49} \mathrm{H}_{84} \mathrm{O}_{21} \mathrm{~N}_{8} \mathrm{~S}_{2}$ for UK1, and $\mathrm{C}_{44} \mathrm{H}_{52} \mathrm{O}_{4} \mathrm{~N}_{2} \mathrm{~S}_{4}, \mathrm{C}_{36} \mathrm{H}_{48} \mathrm{O}_{7} \mathrm{~N}_{8} \mathrm{~S}_{3}, \mathrm{C}_{29} \mathrm{H}_{56} \mathrm{O}_{4} \mathrm{~N}_{10} \mathrm{~S}_{6}, \mathrm{C}_{13} \mathrm{H}_{40} \mathrm{O}_{11} \mathrm{~N}_{26} \mathrm{~S}_{2}$, and $\mathrm{C}_{29} \mathrm{H}_{50} \mathrm{O}_{15} \mathrm{~N}_{7} \mathrm{~S}_{2}$ forUK2, as SBD-derivatized and univalent ion forms. Identification of these unknown compounds should uncover the differences in sulfur metabolism and the antioxidation system between human and mouse, which is a model animal frequently employed for bio thiol-related studies. Further investigation will be required to identify the unknown compounds and clarify their biological roles. 


\section{Conclusions}

An analytical method for bio thiols in mouse sera using an amide-type HILIC column and SBD-F as a thiol-selective fluorogenic reagent was developed. Five bio thiols-Hcy, Cys, CysGly, GSH, and $\gamma$ GluCys - were successfully quantified as total thiol concentrations in the mouse serum samples; the balance between these compounds could be indicative of oxidative stress in the body. This method could be advantageousin the investigation of endogenous sulfur metabolism, since there are few analytical methods that can quantify multiple biothiols, including $\gamma$ GluCys, in biological fluids, and only this method could separate the biothiols and the two unknown peaks in the mouse serum samples. HILIC-q-Orbitrap-MS was also conducted to determine the exact masses of the two unknowns found in the chromatograms of the mouse samples. These exact masses were determined as 1184.519 and 800.281 . Since the unknown compounds had peak intensities comparable to the known biothiols, it is possible that they might function as reductants against oxidative stress in the mouse body. Furthermore, the common product ion, $[\mathrm{SBD}-\mathrm{SH}]^{-}(\mathrm{m} / z$ 230.954), was found in the MS/MS spectra of all the known SBD-biothiols and the two unknowns in the ESI negative mode, which should be useful in thiol metabolomics for confirming that the precursor ions of interest actually bear thiol moieties.

\section{Acknowledgements}

The authors acknowledge their sincere gratitude to Yayoi Kanbayashi, Kenzo Nomura, Daigo Iwahata, Dr. Toshimi Mizukoshi, Dr. Naoyuki Yamada, and Dr. Hiroshi Miyanoat Ajinomoto Co., Inc. This research was partially supported by the Japan Society for the Promotion of Science (JSPS), Grant-in-Aid for Scientific Research (C) (Grant Number 2646033), the Center of Innovation Program from the Japan Science and Technology Agency (JST), and a Grant-in-Aid for scientific research of JSPS Fellows (to M.I., Grant Number 15J12103). 


\section{Figure legends}

\section{Figure 1}

Chemical structures of thiols and SBD-F.

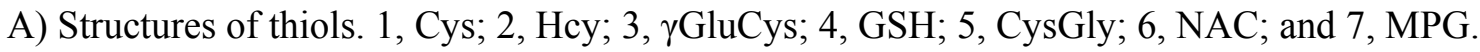

B) Derivatization reaction of cysteine and SBD-F.

\section{Figure 2}

Effect of ammonium formate buffer concentration in the mobile phase on the retention times of SBD-thiols and unknown peaks.

Symbols: $\square$, UK1; $\bullet$, SBD-Hcy; $\Delta$, SBD-Cys; •, SBD-CysGly; $\diamond$, UK2; $\boldsymbol{\Delta}$, SBD-GSH; and $\bigcirc$, SBD- $\gamma$ GluCys. Column: Inertsil Amide $(3.0 \times 150 \mathrm{~mm}, 5 \mu \mathrm{m})$. Column temperature: $35^{\circ} \mathrm{C}$. Mobile phase: acetonitrile-ammonium formate buffer $(\mathrm{pH} 3.0)(75: 25, \mathrm{v} / \mathrm{v})$. Flow rate: $0.4 \mathrm{~mL} / \mathrm{min}$. SBD-MPG and -NAC are omitted, since they were always separated from the other peaks.

\section{Figure 3}

Chromatograms of SBD-thiols:A) in standard sample, B) in mouse serum sample, and C) in human plasma sample.

Peaks: 1, SBD-MPG; 2, SBD-NAC; 3, UK1; 4, SBD-Hcy; 5, SBD-Cys; 6, SBD-CysGly; 7, UK2; 8, SBD-GSH; and 9, SBD- $\gamma$ GluCys. Column: Inertsil Amide $(3.0 \times 150 \mathrm{~mm}, 5 \mu \mathrm{m})$. Column temperature: $35^{\circ} \mathrm{C}$. Mobile phase: acetonitrile-40 $\mathrm{mM}$ ammonium formate buffer $(\mathrm{pH}$ 3.0) (75:25, v/v). Flow rate: $0.4 \mathrm{~mL} / \mathrm{min}$.

\section{Figure 4}

Extracted chromatograms obtained from HILIC-SIM analysis of the SBD-derivatized mouse serum sample. 
A) SBD-Hcy, B) SBD-Cys, C) SBD-CysGly, D) SBD-GSH, E) SBD- $\gamma$ GluCys, F) divalent ion of $\mathrm{UK} 1, \mathrm{G})$ univalent ion of UK1, and H) univalent ion of UK2. Mass tolerance: target mass $\pm 5 \mathrm{ppm}$. Chromatographic conditions were the same as described for Figure 3.

\section{Figure 5}

MS/MS spectra of A) UK1 and B) UK2.

Inset: estimated structure of the common product ion[SBD-SH] $]^{-} m / z$ 230.954. Precursor ions: A, 591.75623 (divalent ion); B, 800.28146 (univalent ion). Collision energies: A, $20 \mathrm{eV}$; B, $30 \mathrm{eV}$. Chromatographic conditions were the same as described for Figure 3. 


\section{References}

[1] A. Meister, Glutathione metabolism and its selective modification, J. Biol. Chem., 263 (1988) 17205-17208.

[2] M.A. Riedijk, B. Stoll, S. Chacko, H. Schierbeek, A.L. Sunehag, J.B. van Goudoever, D.G. Burrin, Methionine transmethylation and transsulfuration in the piglet gastrointestinal tract, Proc. Natl. Acad. Sci. U.S.A., 104 (2007) 3408-3413.

[3] F. Binkley, V. du Vigneaud, The formation of cysteine from homocysteine and serine by liver tissue of rats, J. Biol. Chem., 144 (1942) 507-511.

[4] L. Oppenheimer, V.P. Wellner, O.W. Griffith, A. Meister, Glutathione synthetase. Purification from rat kidney and mapping of the substrate binding sites, J. Biol. Chem., 254 (1979) 5184-5190.

[5] F. Binkley, K. Nakamura, Metabolism of glutathione; hydrolysis by tissues of the rat, J. Biol. Chem., 173 (1948) 411-421.

[6] A.E. Mutlib, J. Shockcor, R. Espina, N. Graciani, A. Du, L.S. Gan, Disposition of glutathione conjugates in rats by a novel glutamic acid pathway: characterization of unique peptide conjugates by liquid chromatography/mass spectrometry and liquid chromatography/NMR, J. Pharmacol. Exp. Ther., 294 (2000) 735-745.

[7] K. Kuśmierek, G. Chwatko, R. Głowacki, P. Kubalczyk, E. Bald, Ultraviolet derivatization of low-molecular-mass thiols for high performance liquid chromatography and capillary electrophoresis analysis, J. Chromatogr. B, 879 (2011) 1290-1307.

[8] S.E. Moriarty-Craige, D.P. Jones, Extracellular Thiols and Thiol/Disulfide Redox in Metabolism, Annu. Rev. Nutr., 24 (2004) 481-509.

[9] M. Kemp, Y.-M. Go, D.P. Jones, Nonequilibrium thermodynamics of thiol/disulfide redox systems: A perspective on redox systems biology, Free Radical Biol. Med., 44 (2008) 921-937.

[10] D.M. Townsend, K.D. Tew, H. Tapiero, Sulfur containing amino acids and human disease, Biomed. Pharmacother., 58 (2004) 47-55.

[11] L.L. Humphrey, R. Fu, K. Rogers, M. Freeman, M. Helfand, Homocysteine level and coronary heart disease incidence: a systematic review and meta-analysis, Mayo Clin. Proc., 83 (2008) 1203-1212.

[12] M.A. Baños-González, E. Anglés-Cano, G. Cardoso-Saldaña, M.A. Peña-Duque, M.A. Martínez-Ríos, B. Valente-Acosta, H. González-Pacheco, A. de la Peña-Díaz, Lipoprotein(a) and Homocysteine Potentiate the Risk of Coronary Artery Disease in Male Subjects, Circ. J., 76 (2012) 1953-1957.

[13] M.C. Puertas, J.M. Martínez-Martos, M.P. Cobo, M.P. Carrera, M.D. Mayas, M.J. Ramírez-Expósito, Plasma oxidative stress parameters in men and women with early stage Alzheimer type dementia, Exp. Gerontol., 47 (2012) 625-630.

[14] S. Zoccolella, C. dell'Aquila, G. Abruzzese, A. Antonini, U. Bonuccelli, M. Canesi, S. Cristina, R. Marchese, C. Pacchetti, R. Zagaglia, G. Logroscino, G. Defazio, P. Lamberti, P. Livrea, Hyperhomocysteinemia in levodopa-treated patients with Parkinson's disease dementia, Mov. Disord., 24 (2009) 1028-1033.

[15] M. Kędzierska, R. Głowacki, U. Czernek, K. Szydłowska-Pazera, P. Potemski, J. Piekarski, A. Jeziorski, B. Olas, Changes in plasma thiol levels induced by different phases of treatment in breast cancer; the role of commercial extract 
from black chokeberry, Mol. Cell. Biochem., 372 (2012) 47-55.

[16] S. Stabler, T. Koyama, Z. Zhao, M. Martinez-Ferrer, R.H. Allen, Z. Luka, L.V. Loukachevitch, P.E. Clark, C. Wagner, N.A. Bhowmick, Serum methionine metabolites are risk factors for metastatic prostate cancer progression, PLoS One, 6 (2011) e22486.

[17] M.Y. Al-Maskari, M.I. Waly, A. Ali, Y.S. Al-Shuaibi, A. Ouhtit, Folate and vitamin B12 deficiency and hyperhomocysteinemia promote oxidative stress in adult type 2 diabetes, Nutrition, 28 (2012) e23-e26.

[18] A. Noce, R. Fabrini, M. Dessì, A. Bocedi, S. Santini, V. Rovella, A. Pastore, M. Tesauro, S. Bernardini, N. Di Daniele, G. Ricci, Erythrocyte glutathione transferase activity: a possible early biomarker for blood toxicity in uremic diabetic patients, Acta Diabetol., 51 (2014) 219-224.

[19] M. Isokawa, T. Kanamori, T. Funatsu, M. Tsunoda, Analytical methods involving separation techniques for determination of low-molecular-weight biothiols in human plasma and blood, J. Chromatogr. B, 964 (2014) $103-115$.

[20] T. Toyo'oka, Recent advances in separation and detection methods for thiol compounds in biological samples, J. Chromatogr. B, 877 (2009) 3318-3330.

[21] M.E. McMenamin, J. Himmelfarb, T.D. Nolin, Simultaneous analysis of multiple aminothiols in human plasma by high performance liquid chromatography with fluorescence detection, J. Chromatogr. B, 877 (2009) 3274-3281.

[22] T.D. Nolin, M.E. McMenamin, J. Himmelfarb, Simultaneous determination of total homocysteine, cysteine, cysteinylglycine, and glutathione in human plasma by high-performance liquid chromatography: Application to studies of oxidative stress, J. Chromatogr. B, 852 (2007) 554-561.

[23] T. Toyo'oka, K. Imai, High-performance liquid chromatography and fluorometric detection of biologically important thiols, derivatized with ammonium 7-fluorobenzo-2-oxa-1,3-diazole-4-sulphonate (SBD-F), J. Chromatogr., 282 (1983) 495-500.

[24] M. Isokawa, T. Funatsu, M. Tsunoda, Fast and simultaneous analysis of biothiols by high-performance liquid chromatography with fluorescence detection under hydrophilic interaction chromatography conditions, Analyst, 138 (2013) 3802-3808.

[25] M. Isokawa, T. Kanamori, T. Funatsu, M. Tsunoda, Recent advances in hydrophilic interaction chromatography for quantitative analysis of endogenous and pharmaceutical compounds in plasma samples, Bioanalysis, 6 (2014) 2421-2439. [26] M. Isokawa, T. Funatsu, M. Tsunoda, Efficient Separation and Sensitive Detection of Biothiols by Hydrophilic Interaction Liquid Chromatography with Fluorescence Detection after Derivatization with 4-Aminosulfonyl-7-fluoro-2,1,3-benzoxadiazole, Chromatography, 35 (2014) 169-172.

[27] M.M. de Rezende, V. D'Almeida, Central and systemic responses to methionine-induced hyperhomocysteinemia in mice, PLoS One, 9 (2014) e105704.

[28] V.C. da Silva, L. Fernandes, E.J. Haseyama, A.L.D.A. Agamme, E.M.G. Shinohara, M.T.C. Muniz, V. D'Almeida, Effect of vitamin B deprivation during pregnancy and lactation on homocysteine metabolism and related metabolites in brain and plasma of mice offspring, PLoS One, 9 (2014) e92683.

[29] C.-C. Hsu, C.-H. Cheng, C.-L. Hsu, W.-J. Lee, S.-C. Huang, Y.-C. Huang, Role of vitamin B6 status on antioxidant defenses, glutathione, and related enzyme activities in mice with homocysteine-induced oxidative stress, Food Nutr. Res., 59 (2015) 25702.

[30] M. Isokawa, T. Funatsu, M. Tsunoda, Evaluation of the Effects of Sample Dilution and Volume in Hydrophilic 
Interaction Liquid Chromatography, Chromatographia, 77 (2014) 1553-1556.

[31] T. Kanamori, T. Funatsu, M. Tsunoda, Evaluation of the Effects of Sample Solutions and Injector Wash Solutions on Separation Efficiency in Hydrophilic Interaction Liquid Chromatography, Chromatography, in Press, DOI: 10.15583/jpchrom.2015.028.

[32] T. Toyo'oka, K. Imai, Fluorescence analysis of thiols with ammonium 7-fluorobenzo-2-oxa-1,3-diazole-4-sulphonate, Analyst, 109 (1984) 1003-1007.

[33] S. Ichinose, M. Nakamura, M. Maeda, R. Ikeda, M. Wada, M. Nakazato, Y. Ohba, N. Takamura, T. Maeda, K. Aoyagi, K. Nakashima, A validated HPLC-fluorescence method with a semi-micro column for routine determination of homocysteine, cysteine and cysteamine, and the relation between the thiol derivatives in normal human plasma, Biomed. Chromatogr., 23 (2009) 935-939.

[34] A. Valente, M.R. Bronze, M. Bicho, R. Duarte, H.S. Costa, Validation and clinical application of an UHPLC method for simultaneous analysis of total homocysteine and cysteine in human plasma, J. Sep. Sci., 35 (2012) 3427-3433.

[35] R. Ferin, M.L. Pavão, J. Baptista, Methodology for a rapid and simultaneous determination of total cysteine, homocysteine, cysteinylglycine and glutathione in plasma by isocratic RP-HPLC, J. Chromatogr. B, 911 (2012) 15-20.

[36] L.-Y. Zhang, F.-Q. Tu, X.-F. Guo, H. Wang, P. Wang, H.-S. Zhang, A new BODIPY-based long-wavelength fluorescent probe for chromatographic analysis of low-molecular-weight thiols, Anal. Bioanal. Chem., 406 (2014) 6723-6733.

[37] A. Zinellu, S. Sotgia, B. Scanu, M.F. Usai, A.G. Fois, V. Spada, A. Deledda, L. Deiana, P. Pirina, C. Carru, Simultaneous detection of N-acetyl-1-cysteine and physiological low molecular mass thiols in plasma by capillary electrophoresis, Amino Acids, 37 (2009) 395-400.

[38] Y. Kono, H. Iizuka, M. Isokawa, M. Tsunoda, H. Ichiba, K. Sadamoto, T. Fukushima, HPLC-fluorescence determination of thiol compounds in the serum of human male and female subjects using HILIC-mode column, Biomed. Chromatogr., 28 (2014) 589-593. 
Table 1

Detection sensitivity and linearity of the developed method

\begin{tabular}{crrrrr}
\hline \multirow{2}{*}{ Thiols } & $\begin{array}{c}\text { LOD }[\mathrm{nM}] \\
\mathrm{S} / \mathrm{N}=3\end{array}$ & $\begin{array}{c}\text { LOQ }[\mathrm{nM}] \\
\mathrm{S} / \mathrm{N}=10\end{array}$ & \multicolumn{2}{c}{$\begin{array}{c}\text { Linearity }[\mathrm{nM}] \\
\mathrm{r}^{2}>0.9999\end{array}$} & \multicolumn{2}{c}{ Regression equation } \\
& slope & intercept \\
\hline NAC & 0.4 & 2 & $5-750$ & 0.001996 & -0.003822 \\
Hcy & 0.6 & 2 & $5-750$ & 0.001477 & -0.003108 \\
Cys & 2 & 7 & $46-6900$ & 0.000376 & -0.003570 \\
CysGly & 0.2 & 0.7 & $5-750$ & 0.003304 & -0.004707 \\
GSH & 0.9 & 3 & $10-1500$ & 0.000882 & -0.002818 \\
$\gamma$ GluCys & 4 & 12 & $15-2250$ & 0.000266 & -0.001452 \\
\hline
\end{tabular}

* $\mathrm{y}$ : relative height compared to SBD-MPG height, $\mathrm{x}$ : SBD-thiol concentration in injection sample [nM] 


\section{Table 2}

Intra-day reproducibility $(n=5)$ and recovery of the developed method in determination of biothiols in mouse serum samples

\begin{tabular}{|c|c|c|c|c|c|}
\hline Thiols & Added $[\mu \mathrm{M}]$ & Measured (mean & SD) $[\mu \mathrm{M}]$ & $\overline{C . V .}[\%]$ & Recovery [\%] \\
\hline \multirow[t]{4}{*}{ Hcy } & 0 & $7.0 \pm$ & 0.07 & 0.9 & - \\
\hline & 15 & $23.3 \pm$ & 0.10 & 0.4 & 108 \\
\hline & 30 & $39.5 \pm$ & 0.11 & 0.3 & 108 \\
\hline & 60 & $71.7 \pm$ & 0.22 & 0.3 & 108 \\
\hline \multirow[t]{4}{*}{ Cys } & 0 & $203.2 \pm$ & 2.09 & 1.0 & - \\
\hline & 75 & $275.8 \pm$ & 1.45 & 0.5 & 97 \\
\hline & 150 & $347.5 \pm$ & 1.61 & 0.5 & 96 \\
\hline & 300 & $497.1 \pm$ & 1.34 & 0.3 & 98 \\
\hline \multirow[t]{4}{*}{ CysGly } & 0 & $1.3 \pm$ & 0.03 & 2.2 & - \\
\hline & 1.25 & $2.5 \pm$ & 0.04 & 1.7 & 95 \\
\hline & 2.5 & $3.7 \pm$ & 0.04 & 1.1 & 97 \\
\hline & 5 & $6.2 \pm$ & 0.12 & 2.0 & 97 \\
\hline \multirow[t]{4}{*}{ GSH } & 0 & $43.3 \pm$ & 0.52 & 1.2 & - \\
\hline & 10 & $54.3 \pm$ & 0.15 & 0.3 & 109 \\
\hline & 20 & $63.8 \pm$ & 0.27 & 0.4 & 102 \\
\hline & 40 & $84.9 \pm$ & 0.39 & 0.5 & 104 \\
\hline \multirow[t]{4}{*}{$\gamma$ GluCys } & 0 & $7.6 \pm$ & 0.13 & 1.8 & - \\
\hline & 5 & $12.9 \pm$ & 0.06 & 0.5 & 107 \\
\hline & 10 & $18.2 \pm$ & 0.11 & 0.6 & 106 \\
\hline & 20 & $28.9 \pm$ & 0.18 & 0.6 & 106 \\
\hline
\end{tabular}


A

1 -

4

6<smiles>CC(=O)NC(CS)C(=O)O</smiles>

B

fig 1<smiles>NC(CS)C(=O)O</smiles>
$\mathrm{NH}_{2}$<smiles>NC(CCS)C(=O)O</smiles><smiles>NC(CCC(=O)NC(CS)C(=O)NCC(=O)O)C(=O)O</smiles><smiles>NC(CCC(=O)NC(CS)C(=O)O)C(=O)O</smiles>

5<smiles>NC(CS)C(=O)NCC(=O)O</smiles>
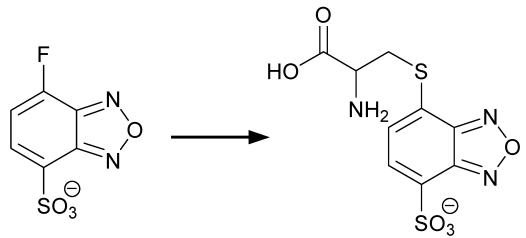


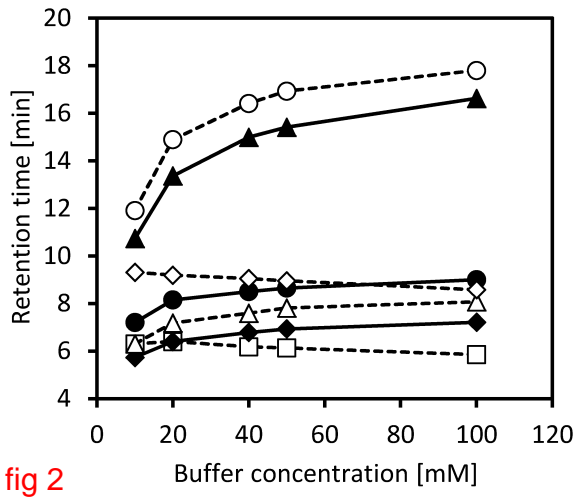



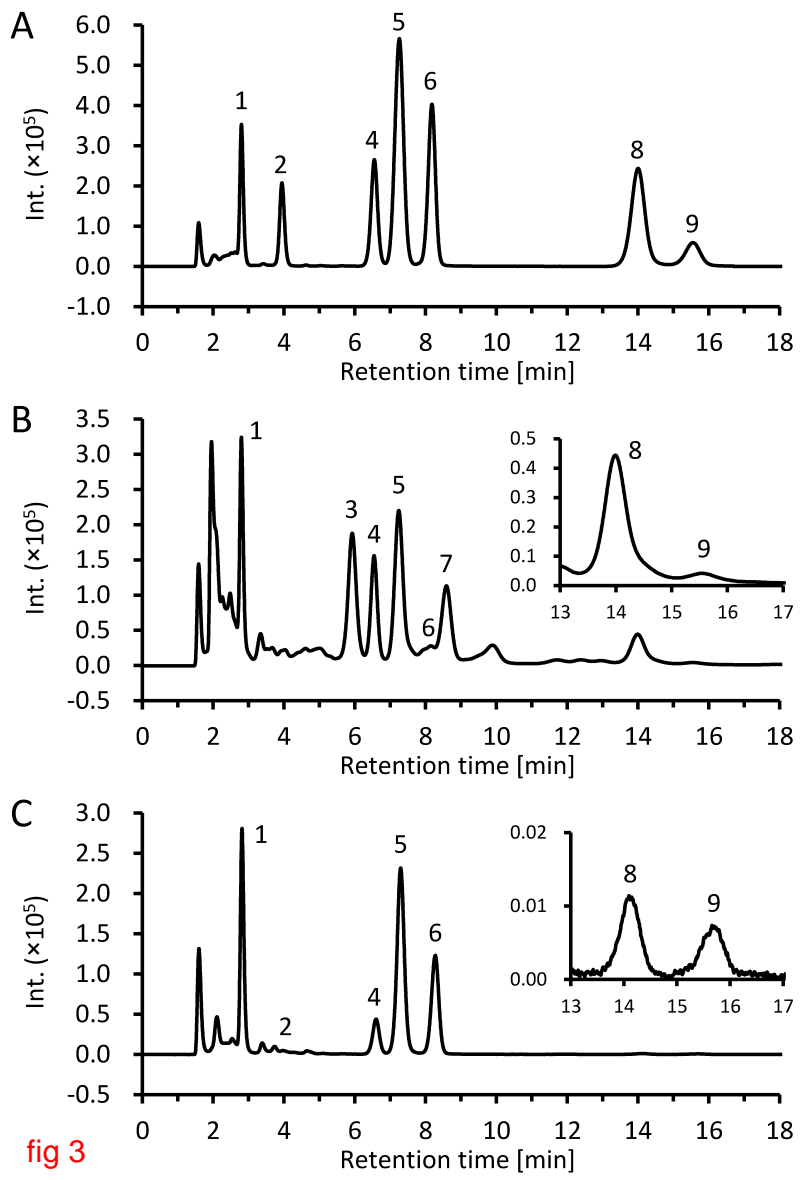


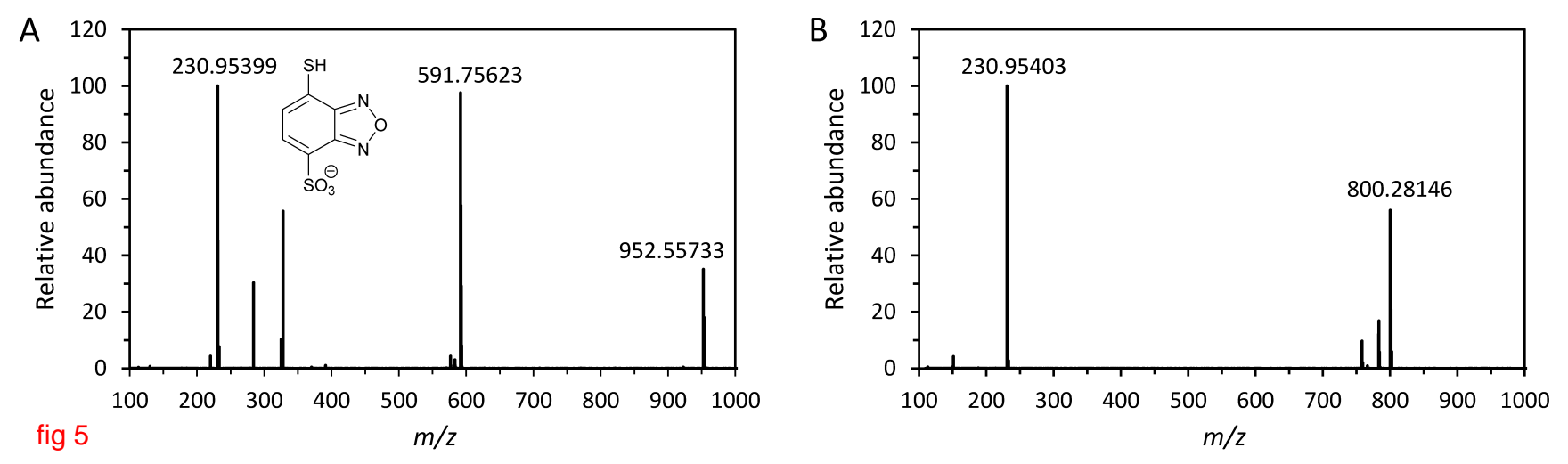

